\title{
Automatisiertes Regenerieren von großen Tiefziehwerkzeugen
}

\author{
Automatyzacja regeneracji dużych narzędzi \\ do głębokiego tłoczenia
}

\section{Streszczenie}

Przeanalizowano problematykę napawania narzędzi do głębokiego tłoczenia. Przedstawiono koncepcję zrobotyzowanego mobilnego stanowiska do napawania z systemem analizy obrazu. Podano przykład zastosowania opracowanego systemu do napawania plazmowego narzędzi, w tym także w pozycjach przymusowych.

\section{Einleitung}

Die Anforderungen an die Unternehmen haben sich in den letzten Jahrzehnten stark gewandelt. Wo früher noch ein Mindestmaß an Stabilität und Kontinuität herrschte, sind heute Dynamik und Diskontinuität an der Tagesordnung. Varianten- und Typenvielfalt bei geringeren Losgrößen fordern eine hohe Flexibilität, um zu einer Verkürzung des Produktlebenszyklus zu gelangen. Die zunehmend verschärfte Wettbewerbssituation der europäischen Länder und insbesondere des Standortes Deutschland im Zuge der fortschreitenden Globalisierung führt dazu, dass viele Unternehmen sowohl die Produktion als auch einen stetig steigenden Anteil an Entwicklungsarbeiten in sogenannte Niedriglohnländer verlagert haben, bzw. verlagern. Hieraus resultiert insbesondere für kleine und mittlere Unternehmen eine äußerst schwierige Marktsituation, in der es im Sinne einer Stärkung der Volkswirtschaft gilt, konkurrenzfähige Alternativen zu schaffen.

Die Robotik ist eine Schlüsseltechnologie für eine wirtschaftliche, marktorientierte und flexible Fertigung. Die Integration in laufende Prozesse von intelligenten,

Prof. Dr.-Ing. habil. Johannes Wilden - Funktionswerkstoffe und Beschichtungen Hochschule Niederrhein, Univ.-Prof. Dr.-Ing. habil. Jean P. Bergmann - Fertigungstechnik Technische Universität IImenau, Niemcy.

\section{Abstract}

The problems of surfacing by welding of deep drawing tools are analyzed in the paper. The concept of a station of surfacing by welding with robot and image analysis system is presented. An example of developed system for plasma surfacing by welding of tools, including the forced positions is shown.

kognitionsorientierten und roboterbasierten Systemen könnte zum Erschließen von neuen Märkten, aber auch zum Sichern und Ausbauen bestehender Märkte führen. Robotersysteme werden für den industriellen Einsatz in der Fertigung zum Teil immer noch wie vor zwanzig Jahren als „freiprogrammierbare Handhabungsautomaten" verstanden. Auf Grund der Entwicklungen in den Assistenz- und Service-Bereichen nimmt die Robotik eine herausragende Stellung als Wachstumstreiber innovativer Märkte ein. Es besteht jedoch immer noch ein erheblicher Bedarf an Forschungsarbeiten, bis vielseitig verwendbare Serviceroboter für verschiedene Alltagsaufgaben einer breiten Anwendungspalette zur Verfügung stehen. Nur durch diese Vorgehensweise werden klein- und mittelständig produzierende Unternehmen in die Lage versetzt, auf Marktschwankungen rasch zu reagieren und an den erforderlichen Veränderungen aktiv teilzunehmen.

Ständig steigende Ansprüche an Produktdesign, Komplexität und technische Reife bei gleichzeitig immer kürzer werdenden Produktlebenszyklen und eine zunehmende Variantenvielfalt stellen immer höhere Anforderungen an den Werkzeug- und Formenbau. Die Endkontur von Formen und Werkzeugen für Blechumformung und Spritzguss wird auch heute noch per Hand hergestellt. Hierzu bringt der Werkzeugmacher seinen umfangreichen persönlichen Erfahrungsschatz ein. Nach dem ersten Tiefziehvorgang wird das Bauteil vermessen und die Toleranzen bewertet (z.B. Rückfederung am Bauteil). Aus dem Soll/lst-Vergleich 
werden dann die Bereiche des Werkzeuges festgelegt, an denen geometrische Änderungen erfolgen müssen. Die Form wird aus der Presse gefahren und die markierten Bereiche werden dann bei Untermaß durch Auftragschweißen bearbeitet. Die aufzutragende Schicht, die in diesem besonderen Fall artgleich ist, beträgt in der Regel 0,1 bis $0,5 \mathrm{~mm}$. Die Handhabung der Form (bis zu 30 t) gestaltet sich auf Grund des hohen Gewichtes als sehr schwierig, so dass das Auftragen in Wannenlage nicht immer möglich ist. Das Handauftragschweißen erfolgt daher in unterschiedlichen Positionen und ist durch eine deutlich höhere Dicke und eine ausgeprägt unregelmäßige Oberfläche gekennzeichnet. Daher werden die bearbeiteten Bereiche nach dem Auftragschweißen spanend bearbeitet, was mit weiteren Kosten und Liegezeiten verbunden ist. Diese aufwendige Verfahrensweise führt sowohl bei der Produktion als auch bei der Instandsetzung zu einem hohen Aufwand und langen Durchlaufzeiten.

Die beim Nachbessern und Regenerieren von Werkzeugen auftretenden Schwierigkeiten wurden schon Ende der achtziger Jahre des letzten Jahrhunderts in Forschungsvorhaben aufgegriffen, konnten aber bis heute nicht zufriedenstellend gelöst werden.

Der bisher aussichtsreichste Ansatz wurde im Rahmen des BMBF-Aufrufes „Forschung für die Produktion von morgen“ durchgeführten Vorhaben „OptoRep“ verfolgt, in dem eine automatisierte Reparaturzelle für den Werkzeugbau entwickelt wurde. Auch wenn dieses System bereits von einem hohen Automatisierungsgrad gekennzeichnet ist, stellt diese Lösung unter den Aspekten der Manipulation, der Kommunikation und der Interaktion mit den Bedienern keine ganzheitliche Vorgehensweise dar. Darüber hinaus sind die Akzeptanz und die Flexibilität der Anlage auch auf Grund der hohen Kosten und der Handhabung von großräumigen Bauteilen sehr gering.

Weitere Lösungsansätze sind in unterschiedlichen Verbunden verfolgt worden, wobei einerseits keine erfolgreiche Lösung für den praxisrelevanten Einsatz des kognitionsorientierten Bearbeitens von verschlissenen Werkzeugen erarbeitet worden ist. Andererseits zeichnet sich die Wahl des Roboters bei diesen Entwicklungen primär als Handhabungssystem, aber nicht als lernfähiges System aus.

Die Robotik zeichnet sich als kostengünstige aber hochwertige Schlüsseltechnologie zur Lösung von fertigungstechnischen Problemen aus. Gelingt es kognitionsorientierte Robotersysteme mit einer einfachen Architektur und einer leistungsfähigen Sensorik für kmU bereit zu stellen, dann ergeben sich über das beantragte Forschungsgebiet hinaus neuartige Marktfelder, welche die Nachhaltigkeit der Entwicklung sicherstellen. Die Entwicklung eines Serviceroboters zur Automatisierung der Werkzeuginstandsetzung erfordert neben der Zusammenführung von Teil- und Einzellösungen auch die Weiterentwicklung der derzeitigen Methoden und Verfahren.
Der aktuelle Stand der Technik belegt die grundsätzliche Eignung verschiedener Technologien um Detailprobleme in der Prozesskette zu lösen. Im Rahmen der Arbeit galt es, die weiterhin bestehenden Defizite

- kein durchgängiges Konzept zum Aufbau eines universellen, intelligenten Servicesystems basierend auf Robotertechnologie (Architektur und Systemdesign)

- keine hocheffiziente und flexible, prozesssiche$\mathrm{re}$, industriell in kmU umsetzbare Fertigungsprozesstechnik (Integration, Anwendungsbezogene Fortschrittevaluierung)

- keine kostengünstige Lösung zur Anpassung leistungsfähiger Sensorik an Roboter, die es ermöglicht, dynamisch Einfluss auf den Auftragschweißprozess zu nehmen

abzubauen und die Robotik für eine branchen- und produktübergreifende Umsetzung in kmU weiter zu entwickeln, sowie als System verfügbar zu machen. Nur so kann die internationale Wettbewerbsfähigkeit gestärkt und ressourcenschonende Produktionsformen eingeführt werden.

\section{Stand der Technik}

Ständig steigende Ansprüche an Produktdesign, Komplexität und technische Reife bei gleichzeitig immer kürzer werdenden Produktlebenszyklen und eine zunehmende Variantenvielfalt stellen höhere Anforderungen an den Werkzeug- und Formenbau. Die Endkontur von Formen und Werkzeugen für Blechumformung und Spritzguss wird auch heute immer noch per Hand hergestellt. Hierzu bringt der Werkzeugmacher seinen umfangreichen persönlichen Erfahrungsschatz ein (Abbildung 1).

Die Herstellungskette eines Umformwerkzeuges kann sehr langwierig und daher sehr kostenintensiv sein. Nach dem ersten Tiefziehvorgang wird das Bauteil vermessen und die Toleranzen bewertet. Aus dem Soll/Ist-Vergleich am Bauteil werden dann die Bereiche des Werkzeuges festgelegt, an denen geometrische Änderungen erfolgen müssen. Die Form wird aus der Presse gefahren und die markierten Bereiche werden dann bei Untermaß durch Auftragschweißen bearbeitet.

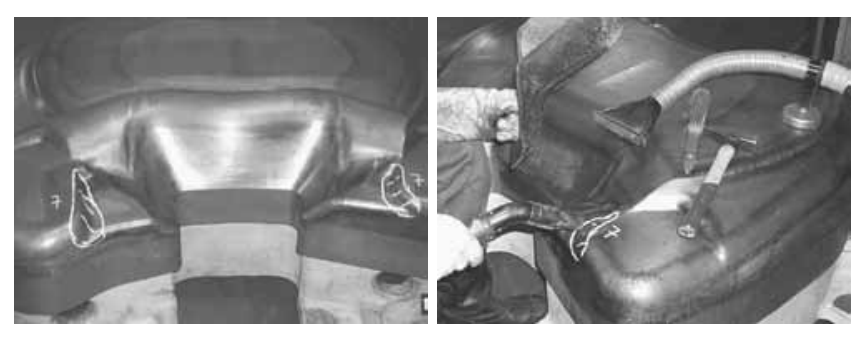

Abb. 1. Beispiel für das Vorgehen des manuellen Auftragschweißens an einer Formfläche ([1]).

Rys. 1. Przykład ręcznego napawania powierzchni formy [1] 


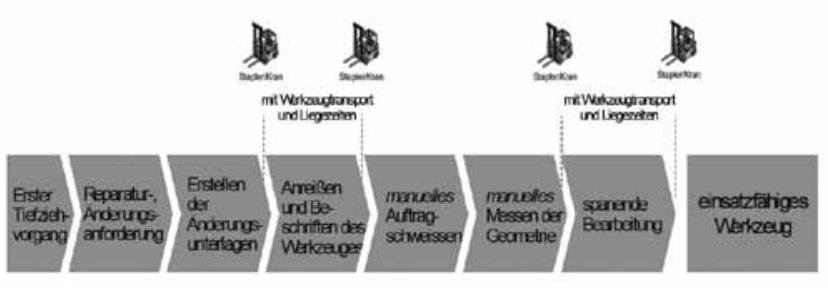

Abb. 2. Heutiges Vorgehen bei dem Bearbeiten von Werkzeugen Rys. 2. Przebieg operacji wytwarzania narzędzi do tłoczenia

Die aufzutragende Schicht, die in diesem besonderen Fall artgleich ist, beträgt in der Regel 0,1 bis $0,5 \mathrm{~mm}$. Die Handhabung der Form (bis zu $30 \mathrm{t}$ ) gestaltet sich auf Grund des hohen Gewichtes als sehr schwierig, so dass das Auftragen in Wannenlage nicht immer möglich ist. Das Handauftragschweißen erfolgt in der Praxis in unterschiedlichen Positionen und ist durch eine stärkere Dicke der Schicht und eine ausgeprägt unregelmäßige Oberfläche gekennzeichnet. Die bearbeiteten Bereiche müssen nach dem Auftragschweißen spanend bearbeitet werden, was mit weiteren Kosten und Liegezeiten verbunden ist. Diese Vorgehensweise führt sowohl bei der Produktion als auch bei der Instandsetzung zu einem hohen Aufwand und langen Durchlaufzeiten (Abbildung 2).

Grundlage für eine Weiterentwicklung, die auf einen kognitionsorientierten, modularen und autonomen Service-Roboter zur Vor-Ort-Instandsetzung von Tiefziehund Spritzgusswerkzeugen abzieht, ist die enge Verknüpfung von Robotik mit Sensorik zum autonomen Erfassen der Arbeitsumgebung und der aktuellen Werkzeuggeometrie, zur kognitionsorientierten Festlegung von Instandsetzungsstrategien. Darüber hinaus spielen die Prozesstechnik zur Instandsetzung und deren Integration in die Anwendung eine hervorgehobene Stellung.

\section{Ansatz und Vorgehensweise}

Abbildung 3 verdeutlicht die Komplexität der Thematik sowie die Vernetzung der einzelnen Arbeitspakete.

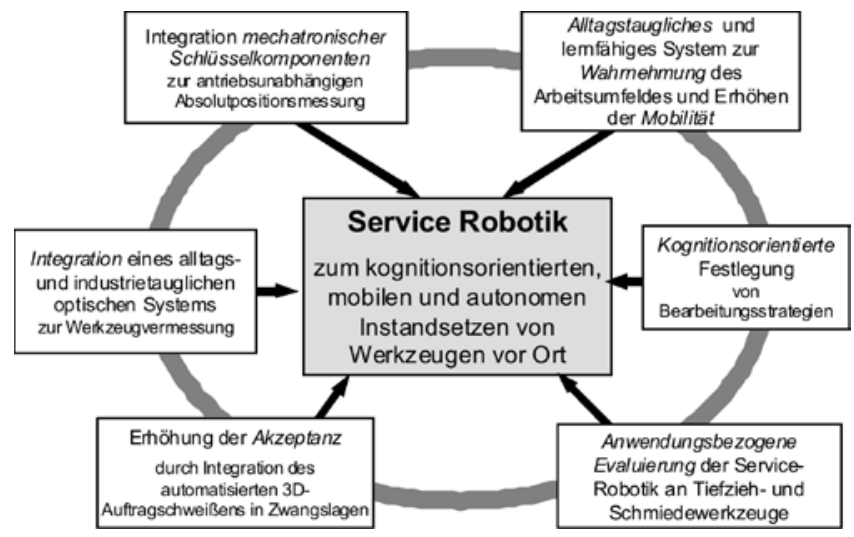

Abb. 3. Arbeitspakete bei der Entwicklung eines kognitionsorientierten, mobilen und autonomen Service-Roboters

Rys. 3. Pakiet zadań przy opracowywaniu mobilnego, automatycznego, współrzędnościowego robota do prac naprawczych
Zur Entwicklung eines kognitionsorientierten, mobilen und autonomen Service-Roboters zur vor Ort Instandsetzung ist die ganzheitliche Betrachtung der Bauteilanforderungen und der Prozessanforderungen unabdingbar.

Der Einsatz der Robotik als Handhabungssystem in der industriellen Fertigung ist bereits in den letzten Jahren erfolgt. Hier werden dem Roboter durch Teach -In Verfahren oder Offline Programmierung die wesentlichen Aufgaben implementiert. Diese Vorgehensweise ist für einen Einsatz im Werkzeugbau jedoch ungeeignet, da die zu bearbeitenden Bereiche zuerst durch erfahrenes Personal erkannt und detektiert werden müssen und komplizierte Konturen durch Teach-In einprogrammiert werden müssen, um dann auftraggeschweißt zu werden. Aus diesem Grund werden derartige Aufgaben heutzutage noch manuell durchgeführt, was zu hohen Maschinenstillstandzeiten führt.

Der Einsatz einer intelligenten und kognitionsorientierter Robotik bietet einen viel versprechenden Ansatz, um Aufgaben auch in wechselnden Umgebungen automatisiert durchführen zu können.

Die zu reparierenden oder modifizierenden Bereiche bei Tiefziehwerkzeugen können bis zu mehreren Metern lang sein, wobei die Höhe im Bereich 0,1 bis $1 \mathrm{~mm}$ sein kann. Die geringe notwendige Dicke stellt hohe Genauigkeitsanforderungen einerseits an die Vermessung und andererseits an die exakte Positionierung im Raum des Roboters sowohl beim Vermessen als auch beim Ausführen. Aktuelle Knickarm Industrierobotersysteme für die Schweißtechnik können diesen Anforderungen nur teils gerecht werden, da die Genauigkeit zwischen 0,1 und 0,5 mm liegt, so dass die Entwicklung einerseits eines sensorintegrierten Methodik zum hochpräzisen Bestimmen der Roboterposition und andererseits eine hochdynamische Erfassung der Formgeometrie notwendig sind.

Das System wird in der Lage sein, durch eine angepasste und leistungsfähige Sensorik und Regelung, einen Soll/lst-Abgleich am eingebauten Werkzeug durchzuführen, die verschlissenen Stellen zu erkennen und durch Auftragschweißen auszubessern.

Eine Grundproblematik der Robotik für die dargestelIte Anwendung liegt darin begründet, dass die hohe Dynamik von derzeitigen Robotern zu Lageabweichungen bei der Bewegung führt. Die sich daraus ergebenden Ungenauigkeiten in Summe sind vergleichbar mit den Abmessungen der zu beschichtenden Bereiche. Somit können die durch die am Roboter angebrachten Messsysteme ermittelten Ergebnisse keine ausreichende Information liefern.

Um hier Abhilfe zu schaffen, wird im Roboter eine antriebsunabhängige Messsensorik integriert, die in einem Regelkreis in Echtzeit die Position des Roboters ermittelt und Korrekturen vornimmt (Dynamische Umgebungserfassung). Tiefzieh- und Spritzgießwerkzeuge verfügen über feste Referenzpunkte, die vom Roboter für die Referenzierung bei wechselnder Aufgabe wahrgenommen werden können. Dadurch wird es 


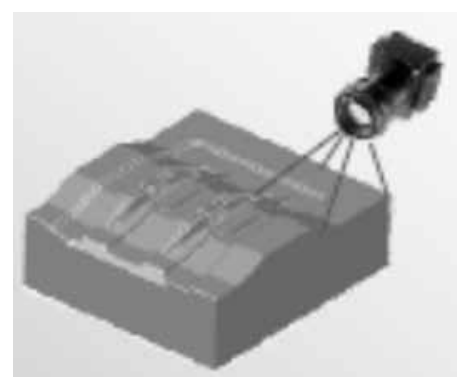

Abb. 4. Schematische Darstellung des Messvorganges (Quelle: ICOM Automation $\mathrm{GmbH}$ )

Rys. 4. Schemat przebiegu pomiaru napoin (źródło: ICOM Automation $\mathrm{GmbH}$ )

möglich sein, den Roboter als mobiles Mess- und Bearbeitungssystem einzusetzen und Aufgaben vor Ort, wie zum Beispiel direkt im Presswerk) durchzuführen.

Das Vermessen erfolgt durch Streifenprojektion. Diese Methode erlaubt es, großräumige Bauteile 3-D mit hoher Genauigkeit mit einer einzelnen Aufnahme zu erfassen (Abbildung 4).

Ein Projektor projiziert Streifen auf das zu untersuchende Objekt und eine Kamera zeichnet das gestreute Licht unter einem Triangulationswinkel auf.

Übliche Streifenprojektionssysteme haben geneigte optische Achsen von Kamera und Projektor - verbunden mit einem räumlich stark abgegrenzten Messfeld entfernt vom Messsystem und Abschattungen bei höheren Objektgradienten [3].

Neueste Entwicklungen zum Aufbau eines kompakten 3-D Systems, basierend auf Streifenprojektion sind in [2] und [3] dargestellt. Das Alleinstellungsmerkmal dieses Systems liegt darin begründet, dass die optischen Achsen parallel zueinander montiert sind, was zu planparallelen Projektions- und Aufnahme-Ebenen führt (Abbildung 5).

Der Abstand der optischen Achsen ist vergleichbar mit dem menschlichen Augenabstand, wodurch auch Objekte mit hohen Gradienten wie z.B. die Innenseite großer Rohre, mit geringen Abschattungen messbar werden. Der Öffnungswinkel von Projektor und Kamera wurde auf mehr als $90^{\circ}$ maximiert. Des Weiteren konnte der Schärfentiefe-Bereich so maximiert werden, dass von ca. $30 \div 40 \mathrm{~cm}$ bis unendlich gearbeitet werden kann.

Durch Überlappung verschiedener Aufnahmen können auch komplizierte Konturen wiedergeben werden. Das entwickelte System wird dann durch Patchworking die Gesamtform des Werkzeuges nachstellen.

Die Informationen aus der Sensorik, aus der Messung und zu dem Auftragschweißen werden von einem lernfähigen System bearbeitet (Abbildung 7). Durch den Soll-/lst-Abgleich und eine spezifische Handlungslogik wird der Roboter in die Lage versetzt eigenständig die Strategie zur Prozessführung beim Auftragschweißen festzulegen. Dieser Zustand wird aus einer Lernphase entstehen, bei dem evolutionär die vom Roboter ausgewählten Strategien durch erfahrenes Bedienpersonal überprüft und ggf. korrigiert werden.

Als Auftragschweißverfahren kommen heutzutage im Verschleißschutz wärmearme Verfahren wie das Plasma-, Laserauftragschweißen oder neuartige Lichtbogenauftragschweißverfahren mit innovativen Stromquellen zum Einsatz. Insbesondere das Plasma-PuIver-Auftragschweißen erscheint für die gegebene
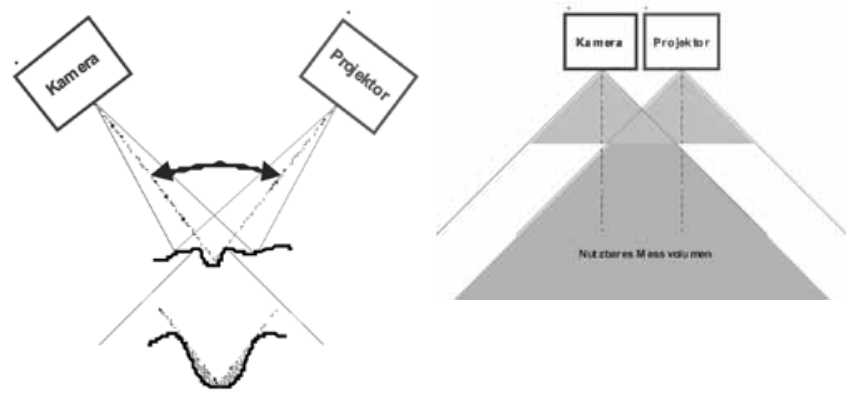

Abb. 5. Links gewöhnliche Streifenprojektionssysteme: begrenztes Messfeld auf Abstand; Abschattungen und rechtsparallele optische Achsen bei kleinem Basisabstand ([2])

Rys. 5. Typowe systemy optyczne: ograniczony obszar pomiarowy (z lewej), równoległe osie optyczne przy małych odstępach bazowych (z prawej) [2]

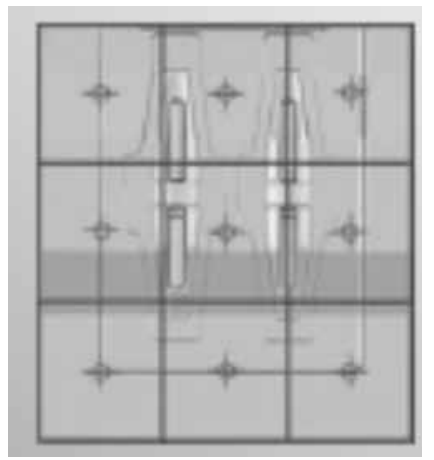

Abb. 6. Patchen mehrerer Aufnahme bei komplizierten Bauteilkonturen (Quelle: ICOM Automation $\mathrm{GmbH}$ ).

Rys. 6. Analiza nałożonych wielu obrazów przy skomplikowanych powierzchniach elementu (źródło: ICOM Automation $\mathrm{GmbH}$ )

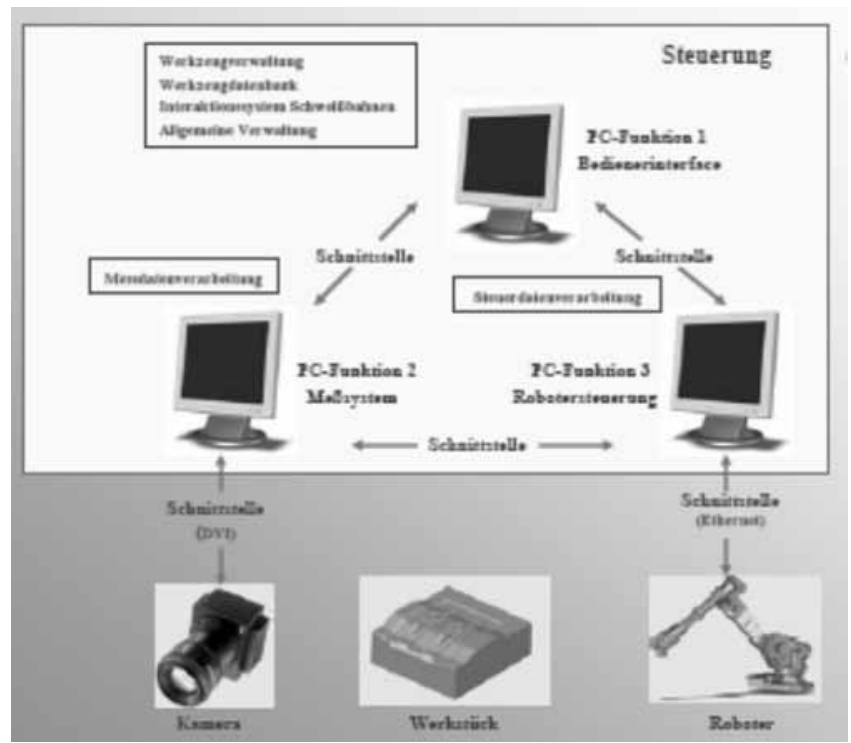

Abb. 7. Schematische Prinzipdarstellung

Rys. 7. Schemat funkcjonowania systemu

Aufgabenstellung, auf Grund der metallurgischen Randbedingungen, besonders geeignet. Hierfür gilt es, das Prozessfeld zum endkonturnahen automatisierten Freiformflächenbeschichten zu parametrisieren.

Darüber hinaus stellt aus schweißtechnischer Sicht die Auslegung des Prozesses unter Berücksichtigung der Bearbeitungsposition eine Schlüsselfunktion dar.

Erste grundlegende Simulationsstudien belegen, dass das Beherrschen der Schmelze beim Bearbeiten in Zwangslagen, zum Beispiel in steigender oder 


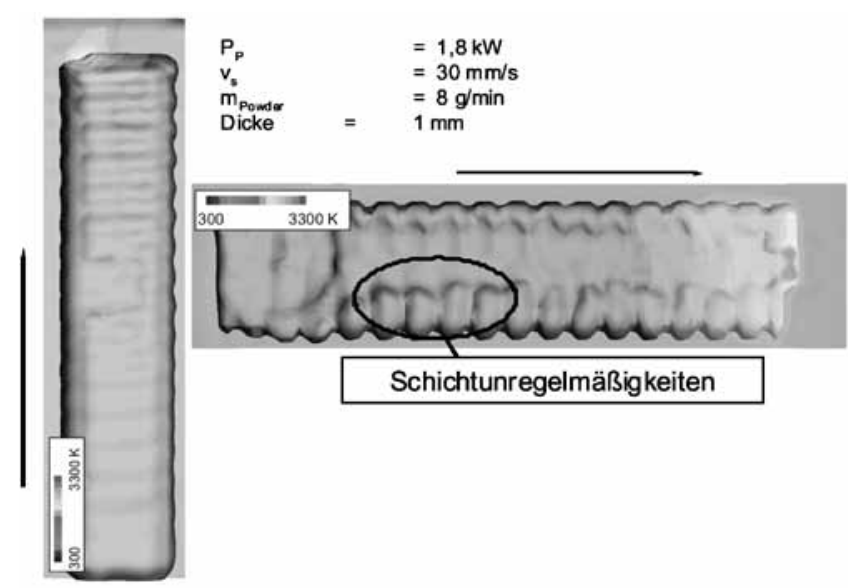

Abb. 8. Simulationsrechnungen zum Auftragschweißen

Rys. 8. Symulacje obliczeniowe napawania

fallend

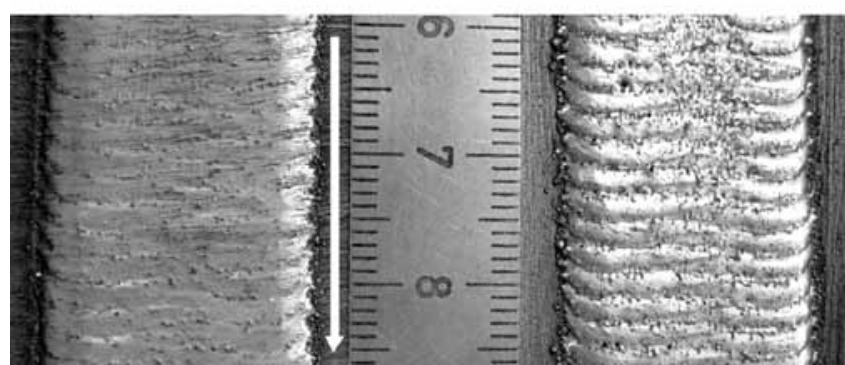

Abb. 9. Experimentelle Untersuchungen

Rys. 9. Badania eksperymentalne
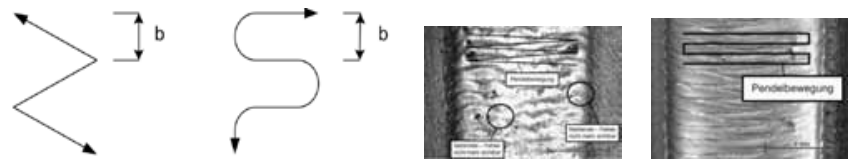

Abb. 10. Einfluss der Bewegung auf die Schichtgüte Rys. 10. Wpływ ruchów zakosowych na jakość warstwy

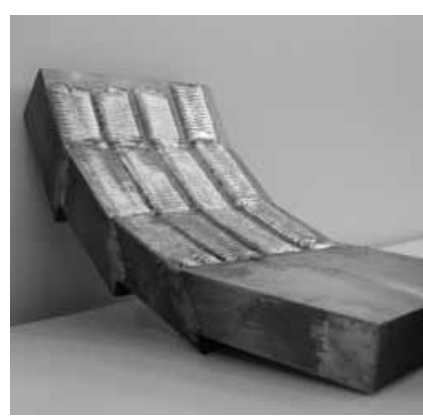

Abb. 11. PTA-Auftragschweißen in Zwangslagen Rys. 11. Napawanie plazmowe w pozycjach przymusowych

fallender Position, erfolgreich durchgeführt werden kann. Abbildung 8 veranschaulicht beispielsweise den Unterschied bei der Schichtbildung zwischen der Wannenlage und dem Auftragschweißen in steigender Anordnung.

Durch einen geringen Wärmeeintrag kann das Schmelzbadvolumen reduziert werden und somit ein Fliessen der Schmelze in Richtung der Erdanziehungskraft vermieden werden.

Eine Überprüfung und Abgleichung der Simulation mit den experimentellen Ergebnissen veranschaulicht 9

Auch die Bewegungsstrategie, die von dem erfahrungsbasierten System entwickelt werden muss spielt eine hervorgehoben Rolle auf die Schichtbildung. So ist eine mäanderförmige Bewegung vorzuziehen, um fehlerfrei Schichten herzustellen (Abbildung 10).

Für das Auftragschweißen in Zwangslagen erfolgt eine strömungsmechanische Auslegung des Brennersystems, die den veränderten Prozessbedingungen Rechnung trägt.

Für eine eventuelle Nachbearbeitung ist es geplant, ein kleinvolumiges Schleifgerät am Endeffektor zu positionieren. Der abzutragende Bereich kann mittels der optischen Messtechnik (Streifenmethode) ermittelt werden und vergleichbar zum Auftragschweißen kann das abzutragende Volumen ermittelt und definiert werden.

\section{Zusammenfassung}

Das Regenerieren von Tiefzieh- und Spritzgus werkzeugen, welches heutzutage üblicherweise händisch durchgeführt wird, stellt vielfältige Anforderungen an die Technologie und ist sehr zeitaufwändig. Automatisierte Lösungen, die mobil an unterschiedlichen Werkzeuggeometrien eingesetzt werden können, sind bisher nicht verfügbar, da keine geeignete Messtechnik, Handlingsysteme, Programmerzeugung und Auftragschweißtechnologie verfügbar ist.

Im Rahmen des Projektes KomoRob wurden Strategien entwickelt, um verschlissene Werkzeuge automatisiert mit hoher Genauigkeit zu vermessen und anhand von vorliegenden CAD Daten ein Fehlermodell zu erstellen. Durch die Erarbeitung eines Sensornetzwerkes in Kombination mit einem Streifenprojektionsmesssystems kann der

Absolutpositionsfehler des verwendeten Roboters analysiert werden, wodurch die Vermessung eines großvolumigen Werkzeuges mit hoher Genauigkeit ermöglicht wurde.

Mittels konventioneller automatisierter Auftragschweißverfahren lassen sich großvolumige Werkzeuge üblicherweise nur in Wannenlage regenerieren. Daher stellte die Weiterentwicklung der PTA-Auftragschweißtechnologie zum Regenerieren von Flächen in Zwangslagen einen weiteren Arbeitsschritt im Rahmen des Projektes dar. Hierzu galt es, die Anlagentechnik entsprechend der Bedingungen zu überarbeiten und geeignete Technologien zum PTA-Auftragschweißen zu entwickeln. Anhand der entwickelten Technologie wurde ein mathematisches Modell aufgestellt, anhand dessen für unterschiedliche 
Bedingungen optimierte Schweißparameter bestimmt werden können.

Ausgehend von den erstellten Fehlermodellen des Werkzeuges erfolgte die automatisierte Programmerstellung zur Regeneration des Werkzeuges. Hierbei werden neben der Schweißbahn auch jeweils optimierte Schweißparameter an die Robotersteuerung, bzw. die Schweißstromquelle übergeben.

Die aufgebaute mobile Bearbeitungsstation beinhaltet alle Komponenten, welche zum automatisierten Regenerieren notwendig sind. Es beinhaltet eine
Kamera zum Messen des Bauteils, einen Schweißbrenner, einen Schleifer zur Endbearbeitung sowie PC-Hardware mit der notwendigen Software zur exakten Positionsbestimmung des Endeffektors, der Erstellung des Fehlermodells und der Programmerzeugung.

Anhand einer umfassenden Evaluierung wurde die Funktionsweise der Einzelkomponenten sowie des gesamten Ablaufs an unterschiedlichen Werkzeugen erfolgreich getestet.

\section{Schrifttum}

[1] Drews, P.; Köhler, G.: Reparatur und Herstellung von technischen Oberflächen an Werkzeugen und Maschinenbauteilen. Shaker Verlag, Aachen, (2003)

[2] Bothe, T., Gesierich, A., von Kopylow, C., Jüptner, W.: 3-DKamera - ein miniaturisiertes Streifenprojektionssystem, Infobörse Mikrosystemtechnik, Heft 43, (2003)

[3] Bothe, T., Li, W., Kopylow, C., Jüptner, W.: High Resolution 3D Shape Measurement on specular surfaces by fringe reflection, Proc. SPIE Int. Soc. Opt. Eng. 5457, (2004), 411-422

\section{Danksagung}

Das Vorhaben ist im Themenfeld „Servicerobotik" angesiedelt und aus Mitteln des Bundesministerium für Bildung und Forschung (BMBF) im Rahmen von „Produktion für morgen“ finanziert und vom Projektträger Forschungszentrum Karlsruhe betreut. Für die Unterstützung sei gedankt.

Mitglieder des Konsortiums sind die Firmen Reis Robotics (Obernburg), Durum GmbH (Willich), ICOM Automation $\mathrm{GmbH}$, AOS GmbH (Dresden), Rui Barradas Schweißtechnik (Dortmund), Bräuer Schweißtechnik GmbH (Kirchhunden), Schwarz Werkzeugbau $\mathrm{GmbH}$, BIAS $\mathrm{GmbH}$ (Bremen), das Fachgebiet Fertigungstechnik der Technischen Universität IImenau und der Lehstuhl für Regelungssystemtechnik der Universität Dortmund.

\section{\$ 4 METAL.PL}

\section{Ponad 2000 podwykonawców z całego świata}

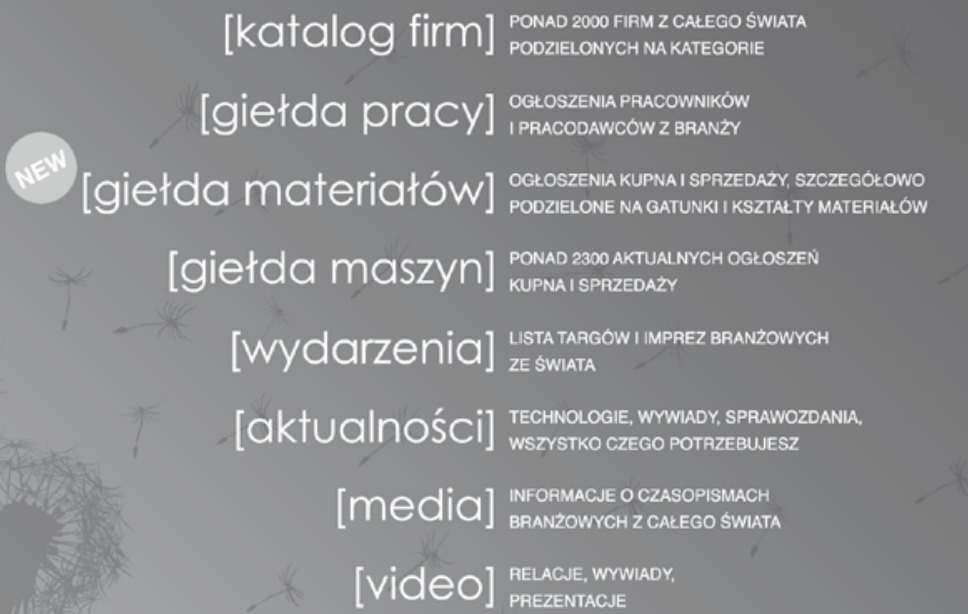

mww.4metal.p

$=$ mun.4metal.de

wnw.4metal.cz

होत्व $w w w .4$ metal.com

$\equiv$ mww.4metal.nl

Emw.4metal.at

III www.4metal.be

Enw.4metal.ru

+ mww.4metal.ch

[I] nww.4metal.it

III wnw.4metal.ro

$\triangleq$ mww.4metal.hu

wnw.4metal.us 